\title{
A CHARACTERIZATION OF COMPACT GROUPS
}

\author{
DAVID L. JOHNSON
}

\begin{abstract}
It is shown that the group algebra $L^{1}(G)$ of a locally compact group $G$ is an ideal in the bidual $L^{1}(G)^{* *}$ of $L^{1}(G)$ (equipped with Arens product) if and only if $G$ is compact.
\end{abstract}

Let $G$ be a locally compact group with group algebra $L^{1}(G)$ and let the bidual $L^{1}(G)^{* *}$ of $L^{1}(G)$ be equipped with an Arens product as in [2]. It is known [5, Corollary 3.4] that, if $G$ is compact, then $L^{1}(G)$ is an ideal in $L^{1}(G)^{* *}$. The converse was asserted in [4, Theorem 7.5]; however, the "proof" given there was based upon a false result (Lemma 7.1 of [4]). In this note, we give a proof of the converse and also show why [4, Lemma 7.1] is false in the generality stated.

THEOREM 1. A locally compact group $G$ is compact if and only if $L^{1}(G)$ is an ideal in $L^{1}(G)^{* *}$.

Proof. We will only establish the implication $(\Leftarrow)$. Let $W(G) \subset L^{\infty}(G)$ be the commutative $C^{*}$-algebra of all continuous weakly almost periodic functions on $G$ (see [1]), and let $G_{W}$ be its maximal ideal space. Then

$$
M\left(G_{W}\right)=W(G)^{*}=L^{1}(G)^{* *} / W(G)^{\perp}
$$

and, because $L^{1}(G)$ is an ideal in $L^{1}(G)^{* *}$, it is also an ideal in $M\left(G_{W}\right)$. Thus, by [3, Theorem 2.1, Corollary 2.3], $M\left(G_{W}\right)=M(G)$ and, as a result, $C_{0}(G)=$ $W(G)$. However, the function 1 is in $W(G)$, so $G$ is compact.

The proof given above was constructed jointly by the author and Charles D. Lahr; it replaces a more elementary, but slightly longer, proof due to the author.

Now, let $A$ be a Banach algebra, let $M_{r}(A)$ be the Banach algebra of all continuous right multipliers of $A$ and let $G_{r}(A)$ be the subset of $M_{r}(A)$ consisting of all isometric onto right multipliers of $A$. Lemma 7.1 of [4] states that, if the closed unit ball of $M_{r}(A)$ is compact in the weak operator topology $\tau_{r}$ it inherits from $\mathbf{B}(A)$, then $\left(G_{r}(A), \tau_{r}\right)$ is a compact topological group. The following theorem shows that this is false even for dual $C^{*}$ algebras $A$ (compare with [4, Theorem 7.4]). For such an $A$, it is known [5, Theorem 2.2] that $M_{r}(A)=A^{* *}$, from which it follows easily that the unit ball of $M_{r}(A)$ is $\tau_{r}$-compact.

Received by the editors May 5, 1978.

AMS (MOS) subject classifications (1970). Primary 22D15, 43A20; Secondary 43A60, 46L05.

Key words and phrases. Group algebra, Arens product, weakly almost periodic function, dual $C^{*}$-algebra. 
TheOREM 2. Let $A=C C(H)$ be the dual $C^{*}$-algebra of all compact operators on a Hilbert space $H$. Then $G_{r}(A)$ is $\tau_{r}$-compact (if and) only if $H$ is finite dimensional.

Proof. First, the bidual $A^{* *}$ of $A$ can be identified with $\mathrm{B}(H)$; thus, $G_{r}(A) \subset \mathbf{B}(H)$. In fact, it is routine to establish that $G_{r}(A)$ is the group $U(H)$ of unitary operators on $H$. Next, let $x, y \in H$ and let $T_{\alpha} \rightarrow T$ in the $\tau_{r}$-topology on $G_{r}(A)$. Let $M$ in $A$ be such that $M x=x$ (if $x \neq 0$, then $M z=\left((z \mid x) /\|x\|^{2}\right) x$ will do), and let $M^{*}$ in $A^{*}$ be defined by $\left\langle N, M^{*}\right\rangle=$ $(N x \mid y)$. Then

$$
\left(T_{\alpha} x \mid y\right)=\left(T_{\alpha} M x \mid y\right)=\left\langle T_{\alpha} M, M^{*}\right\rangle \rightarrow\left\langle T M, M^{*}\right\rangle=(T M x \mid y)=(T x \mid y),
$$

so $T_{\alpha} \rightarrow T$ in the weak operator topology $\tau_{\text {wo }}$ on $\mathbf{B}(H)$. Therefore, if $G_{r}(A)$ is $\tau_{r}$-compact, then $U(H)$ is $\tau_{\text {wo }}$-compact, and this cannot occur if $H$ is infinite-dimensional.

ADDED IN PROOF. It has recently come to my attention that Theorem 1 was proved, using different methods, by S. Watanabe [A Banach algebra which is an ideal in the second dual space, Sci. Rep. Niigata Univ. 11 (1974), 95-101].

\section{REFERENCES}

1. R. B. Burckel, Weakly almost periodic functions on semigroups, Gordon and Breach, New York, London, Paris, 1970.

2. P. Civin and B. Yood, The second conjugate space of a Banach algebra as an algebra, Pacific J. Math. 11 (1961), 847-870.

3. S. A. McKilligan, On the representation of the multiplier algebras of some Banach algebras, J. London Math. Soc. (2) 6 (1973), 399-408.

4. B. J. Tomiuk, Multipliers on Banach algebras, Studia Math. 54 (1976), 267-283.

5. P.-K. Wong, On the Arens product and annihilator algebras, Proc. Amer. Math. Soc. 30 (1971), 79-83.

Department of Mathematics, Dartmouth College, Hanover, New Hampshire 03755

Current address: Department of Mathematics, University of Southern California, Los Angeles, California 90007 